Rabaska

Revue d'ethnologie de l'Amérique française

\title{
Marius Barbeau et la danse
}

\section{Pierre Chartrand}

Volume 13, 2015

Présence de Marius Barbeau : l'invention du terrain en Amérique française. Autour d'un legs centenaire (1914-2014)

URI : https://id.erudit.org/iderudit/1033761ar

DOI : https://doi.org/10.7202/1033761ar

Aller au sommaire du numéro

Éditeur(s)

Société québécoise d'ethnologie

ISSN

1703-7433 (imprimé)

1916-7350 (numérique)

Découvrir la revue

Citer cet article

Chartrand, P. (2015). Marius Barbeau et la danse. Rabaska, 13, 188-197.

https://doi.org/10.7202/1033761ar

Ce document est protégé par la loi sur le droit d'auteur. L'utilisation des services d'Érudit (y compris la reproduction) est assujettie à sa politique d'utilisation que vous pouvez consulter en ligne.

https://apropos.erudit.org/fr/usagers/politique-dutilisation/ 


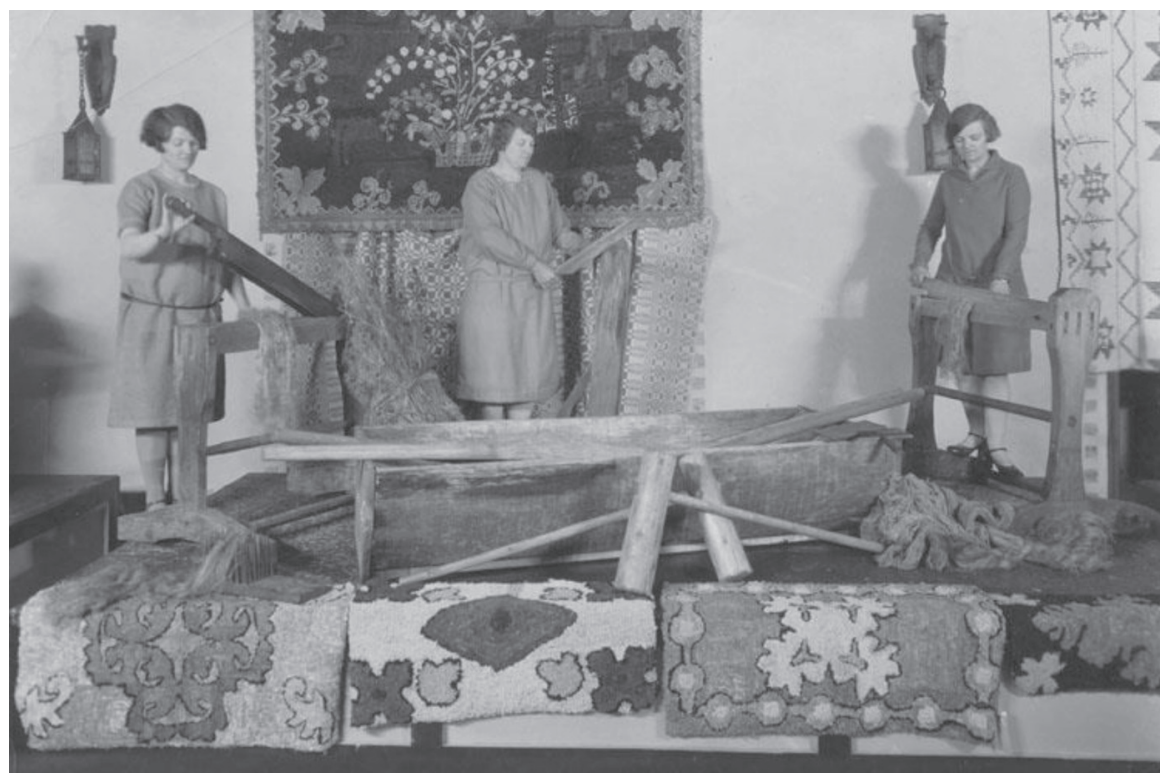

Festival de la chanson et des métiers du terroir au Château Frontenac, Québec, 1928.

$\mathrm{M}^{\text {me }}$ Pouliot et $\mathrm{M}^{\text {mes }}$ Leblond utilisant des machines à couteaux pour séparer et nettoyer le lin («brayeuse ») et un foulon en avant-plan. Source : Musée canadien de l'histoire n B563-5.7. 


\section{Marius Barbeau et la danse}

Pierre Chartrand

Centre Mnémo, Drummondville

Quelle place la danse a-t-elle occupée dans la vie de Marius Barbeau, tant dans ses recherches et collectes que dans ses activités de " programmateur » d'événements de mise en valeur du patrimoine ? Nous commencerons par faire un inventaire de ses collectes en danse ou de ce qu'il a publié sans nécessairement l'avoir collecté lui-même. Cet inventaire ne prétend aucunement à l'exhaustivité. Il nous donnera cependant une bonne idée de l'importance que la danse a pu prendre dans le cadre de son travail.

\section{Collectes publiées}

Les travaux en danse de Marius Barbeau se concentrent essentiellement sur les rondes chantées. Nous présentons ci-dessous l'inventaire de ce qu'il a publié dans En roulant ma boule ${ }^{1}$, en donnant les commentaires succincts qu'il fait en bas de page, puisque partition et paroles représentent l'essentiel des informations données. C'est dans ces commentaires que nous risquons de trouver quelques informations sur la danse. Nous donnerons ensuite son principal livre portant sur les rondes, soit Dansons à la ronde / Roundelays ${ }^{2}$

\section{En roulant ma boule}

Nous n'avons retenu ici que la section « Rondes » (p. 405-482) puisque ce n'est qu'ici que Barbeau traite de danse. Cette section comporte 37 pièces. La section précédente « Fredons de danse » (p. 319-402) ne fait que l'analyse des chansons. Nous n'en avons donc pas tenu compte. Les pièces sont présentées en ordre alphabétique. Nous donnons le numéro de page et la numérotation de Barbeau pour chaque citation.

1. Marius Barbeau, En roulant ma boule. Deuxième partie du Répertoire de la chanson folklorique française du Canada, [Ottawa], Musée national de l'Homme/Musées nationaux du Canada, Ottawa, 1982, xxvIII-753 p.

2. Marius Barbeau et al., Roundelays / Dansons à la ronde, Ottawa, Ministère du Nord canadien et des ressources nationales, Musée national du Canada, «Bulletin » ${ }^{\circ} 151, \mathrm{n}^{\circ} 41$ de la série anthropologique, 1958, $104 \mathrm{p}$. 
Avoine, avoine ! : « Les enfants chantent et dansent cette ronde en se tenant par la main. Après les deux premiers vers, ils s'arrêtent et ils imitent, suivant le cas, les gestes du laboureur, du semeur, du faucheur, du moissonneur, du batteur, etc. Au vers suivant, pour marquer le temps, ils se croisent les bras sur la poitrine, en disant : "Comme ceci", et ils se les croisent en sens inverse en disant: "Comme cela". Puis, en accompagnant les mots, ils tapent du pied, se frappant les mains : au dernier vers, ils se prennent bras dessus bras dessous en se faisant face et ils font un tour sur eux-mêmes. Ils recommencent à chaque couplet. » (p. 447, n¹72)

Bonhomme, que sais-tu donc faire ? : « [...] Ainsi, les danseurs à l'unisson frappent la terre du pied, des mains, des coudes, puis ils se relèvent et dansent vivement aux mots rythmés du refrain : Terre, terre, terre...» (p. 455-456, nº 175)

C'est la plus belle de céans : «Elle n'est pas, comme Qu'est-ce qui passe ici, si tard?, une ronde d'enfants, mais plutôt d'adultes, comme on le reconnaît aux baisers convoités qu'elle suggère sans doute. [...] Cette ronde se danse en cercle, suivant la description que m'en a donnée Adélard Lambert. Les jeunes danseurs tournent en rond. "La plus belle" brise le cercle et va passer sous les bras joints des voisins, levés en forme d'arc sur son passage ; elle entraîne par la main à sa suite toute la chaîne. À mesure que la chaîne défile sous les bras arqués, elle se referme, mais cette fois la chaîne est retournée et les danseurs ont la face hors du cercle. Une autre "plus belle", la voisine, brise le cercle à son tour, pour l'entraîner sous de nouveaux bras arqués et ramener la file indienne à sa première formation, face en dedans. Toutes les danseuses deviennent à tour de rôle "la plus belle". Il ne peut donc y avoir de jalouse. La ronde se continue au bon plaisir des danseurs, et le refrain se répète assez longtemps pour permettre à toute la chaîne de se refermer. » (p. 422, n 60)

C'est mon beau laurier: «Dans cette ronde, les danseurs adultes (Il ne s'agit pas d'un jeu d'enfants !) font demi-tour dans un sens et demi-tour dans l'autre, en chantant le couplet en solo, et le refrain en chœur. Le solo, de même que le baiser, viennent de celui qui, au début, s'est emprisonné dans le cercle. Après avoir tourné trois fois, les danseurs acceptent le choix que le prisonnier fait d'une compagne, le reçoivent dans leurs rangs, tandis que le danseur suivant dans la file se renferme à son tour dans le cercle. » (p. 415, $\left.\mathrm{n}^{\circ} 157\right)$

Grand cordonnier : "À ces mots, le cordonnier court après les demoiselles. Celle (ou celui) qu'il attrape prend sa place et devient "grand cordonnier" ; et la ronde reprend. $\gg\left(\right.$ p. $\left.465, \mathrm{n}^{\circ} 180\right)$

J'ai un beau château : " Pour chanter cette ronde, les enfants, formant deux groupes, tournent en cercle, en se donnant la main. Le premier comprend deux enfants, et le second, tous les autres. À la mention du nom de l'un d'entre eux, les chanteurs du premier groupe vont le chercher et celui-ci se joint à leur cercle. La ronde recommence alors au premier couple, puis on la termine en nommant 
un autre chanteur ; et ainsi de suite jusqu'à ce qu'il ne reste plus d'enfants dans le second groupe. » (p. 467, $\left.\mathrm{n}^{\circ} 181\right)$

Le clairon du roi joli : «[...] les participants se mettent en cercle et se passent un objet de main à main, alors qu'une personne placée au centre du cercle essaie de deviner où l'objet est rendu. On chante cette chanson au cours du jeu.» (p. 473, n 184)

La tour, prends garde! : « Cette ronde dialoguée comprend trois groupes de chanteurs. Le premier, A, se compose de deux chanteurs, les ennemis de la tour ; le second, B, comprend le duc et ses deux gardes ; et le dernier, C, qui est celui de la tour, réunit le reste des enfants. Le duc est assis sur un trône improvisé, flanqué, à droite et à gauche, de ses deux gardes. Les chanteurs des deux autres groupes se tiennent par la main et forment deux cercles, qui tournent en chantant. $\mathrm{Au} 5^{\mathrm{e}}$ couplet, les ennemis vont lentement s'agenouiller devant le duc, à qui ils font une révérence. Au $8^{\mathrm{e}}$ couplet, le duc envoie des gardes, qui, après lui avoir fait une révérence, vont à la tour, prennent un de ses membres et vont le remettre au premier groupe. Puis la ronde reprend jusqu'à ce que tous les chanteurs de la tour aient passé aux ennemis. (p. 469, n 182)

Marion danse : «Ronde à baisers qui se répète indéfiniment, chantée par Édouard Hovington... » (p. 419, $\left.\mathrm{n}^{\circ} 159\right)$

Le nid du lièvre : « Ronde à baisers... » (p. 437, $\left.\mathrm{n}^{\circ} 167\right)$

Qu'est-ce qui se passe ici, si tard? : « Dans cette ronde, une rangée de jeunes filles et une rangée de garçons se font face. Les danseurs avancent et reculent en chantant tour à tour, en dialoguant. Au mot : "Choisissez !", un garçon choisit une fille, et la ronde recommence. » (recueillie par É.-Z. Massicotte auprès d'Adélard Lambert) (p. 425, $\mathrm{n}^{\circ} 161$ )

\section{Autres danses sans aucun commentaire portant sur la danse}

Ah, toi belle hirondelle ; Allons au bois! ; Bon cordonnier ; Ce n'est pas encor' ici; Ce sont les dames de Rouen; Dans ma main droite; Hier au soir, j'ai été danser; J'ai tant d'enfants à marier; J'ai un pied qui remue; J'ai vu la boulangère ; La bistringue; Laquelle marierons-nous I ? ; Laquelle marierons-nous II ? ; On n'ira plus au bois ; Où vas-tu, boiteux l'ermite? ; Petit pétampon ; Petit pompon; Pierrot n'a pas de chapeau; Quand la boiteuse; Quand la boiteuse s'en vat au bois; Qui veut manger du lièvre I; Qui veut manger du lièvre II ; Savez-vous planter des choux? ; Trois fois passera ; Où vas-tu, mon petit garçon? ; Quel est ton nom?

Nous avons donc onze danses avec commentaires, certains fort brefs, et vingt-six autres sans aucune remarque relative à la danse. Certains commentaires ne présentent qu'une catégorie (chansonnette de danse, ronde à baiser) sans qu'on ait développé ce type de catégorie. Qu'est-ce qu'une chansonnette de danse ? Est-ce une danse chantée tout du long ? Un appel chanté ne durant 
que quelques mesures au début de la danse ? Ou autre chose ? Est-ce que la ronde à baiser ne serait réservée qu'aux adultes, comme Barbeau semble le suggérer dans son commentaire sur C'est mon beau laurier ? C'est ce qu'il semble suggérer dans C'est la plus belle de céans ${ }^{3}$. Il y donne cependant beaucoup de détails sur la provenance de la chanson et des paroles.

Quant à l'analyse du mouvement corporel, ces rondes-jeux (sans pas spécifiques) ne s'y prêtent guère. On aurait cependant pu développer un peu sur les déplacements dans l'espace. On perçoit bien que les remarques ne visent pas la reproduction du mouvement, mais cherchent plutôt à décrire la « fonction » de telle ou telle ronde, à donner l'argument du jeu en question.

\section{Dansons à la ronde / Roundelays}

Cette publication est dédiée « À feu Adélard Lambert, chanteur et folkloriste né à Berthier-en-haut, domicilié en Nouvelle-Angleterre, de retour en sa vieillesse à son foyer natal. La plupart de ces rondes et de leurs airs sont de lui. » (p. 6) Barbeau agit donc grandement en tant qu'éditeur des collectes de Lambert. Il poursuit plus loin :

Quant à la manière de danser ou de jouer ces rondes, il n'a pas été possible d'obtenir beaucoup de renseignements parce qu'elles provenaient de vieux chanteurs qui les considéraient comme des « souvenirs du temps passé »ou « d'en premier» (ce dernier terme vient de Cadie). Mais partout où la tradition française ou même européenne de danser et de jouer a survécu en Amérique, il sera facile à la jeunesse de langue française ou anglaise d'en reconstituer et d'en faire revivre le rythme et les gestes, qui tiennent d'ailleurs d'une réinterprétation vivante et constamment renouvelée. » (p. 96-97)

Ce paragraphe démontre l'intérêt que porte Barbeau à la transmission, dont il a une idée très « moderne » tant elle se rapproche de la vision contemporaine de l'UNESCO, par la phrase : « réinterprétation vivante et constamment renouvelée ».

\section{Les danses de Dansons à la ronde}

Voilà la liste alphabétique des rondes de cette publication. Plusieurs se retrouveront dans En roulant ma boule, qui paraîtra deux décennies plus tard.

Avoine, avoine! ; Bien travailler ; Boiteux d'hermite ; Bon cordonnier ; Bonhomme, bonhomme; Cassons les oufs; Chanson des fleurs ; Couvre-feu; Fraises du bois joli ; La boiteuse au marché; Le pâté de Rouen; Lundi, jour de lavage; Michaud a tombé ; Mon oncle, mon oncle; Omelette au lard; Petite boiteuse ; Petite hirondelle; Petite souris grise ; Petites Dorionnes ; Ronde du loup ; Sur le pont d'Avignon.

3. Il écrit : «Une ronde d'enfants, mais plutôt d'adultes, comme on le reconnaît aux baisers convoités qu'elle suggère sans doute $»$. 
Il n'y a que Bonhomme, bonhomme qui donne un peu plus d'information :

Elle incorpore deux types très anciens de chants populaires de l'Europe, de la France en particulier : une danse à pas très vifs, comme ceux de la gigue ou du reel, et une rengaine dans le style de «Que donnerai-je à m'amie ? » [...] Le morceau se danse comme il se chante, avec des pas gigués et des gestes imitatifs. (p. 98-99)

Là encore on aurait aimé en connaître un peu plus sur le contenu moteur des « pas très vifs ». Qu'entend Barbeau par ses " pas gigués » ? Sont-ils frottés (shuffle), percussifs ? Cela ne semble guère probable dans le cadre d'une ronde pour enfant.

\section{Les Veillées du bon vieux temps ${ }^{4}$ (18-19 mars 1919)}

Parallèlement à ses recherches et collectes, Marius Barbeau est aussi actif comme organisateur et programmateur d'événements liés au patrimoine. C'est ainsi qu'il présentera ses fameuses Veillées du bon vieux temps au Monument national, à Montréal, en 1919 et 1920, et qu'il sera impliqué dans les Festivals de la Chanson et des Métiers du terroir, sous l'égide du Canadien Pacifique à Québec, en 1927 et $1928^{5}$. Ces deux festivals auxquels participa Barbeau ne donnent pas d'informations sur la danse. Nous ne ferons donc pas l'analyse de leurs programmes.

Analysons donc les commentaires publiés dans les programmes des Veillées du bon vieux temps portant sur la danse.

\section{Le 18 mars 1919 (numéros dansés)}

En 9) GIGUE SIMPLE. Exécutée par M. F.-X. Baulne, accompagnée par M. Ménard Bougie. p. 90 : M. Beaulne, qui va répéter une « gigue simple » ou un « reel »... noté en $2 / 4$.

La " gigue simple » de la page 92 est également en 2/4. Suivi de SaintAlbans jig, définie comme gigue double, et notée en doubles croches comme la précédente. On peut donc se demander ce qu'entend Barbeau par « gigue simple ». Ça ne semble pas être la fameuse mélodie éponyme qui est strictement liée à la gigue, mais qui est constituée d'une mesure à trois à division binaire (3/2). L'équivalence " gigue simple » ou un « reel » confirme le fait. Qu'entend-il par ailleurs par " gigue double»? L'appellation fait-elle référence à la musique ou aux pas ? Ce sont des appellations qu'on retrouve fréquemment dans la langue populaire, sans qu'y soit associé un sens précis.

4. Marius Barbeau, « Préface » dans Veillées du bon vieux temps à la Bibliothèque Saint-Sulpice, à Montréal les 18 mars et 24 avril 1919. Sous les auspices de La Société historique de Montréal et de La Société de Folklore d'Amérique (Section de Québec), Montréal, G. Ducharme, Libraire-éditeur, 1920.

5. Merci à Benoît Thériault de m'avoir indiqué que Marius Barbeau n'avait pas participé au $3^{\mathrm{e}}$ Festival de la Chanson et des Métiers du terroir (1930). 
Barbeau ne dissipe pas le flou qui les entoure.

M. Bougie avait auparavant interprété LA GATINEAU (Gigue double), et LE RÊVE DU DIABLE (cotillon).

Pourquoi qualifie-t-on le Rêve du diable de cotillon ? Aucune définition de cotillon n'est donnée ailleurs dans le programme (ni de gigue simple ou double).

Et M. Beaulne avait joué (en 8) LA FRÉTILLANTE (gigue simple) et le MONEYMUSK (reel écossais). (p. 90, il mentionne « le reel » «à la canadienne»).

\section{Le 24 avril 1919 (numéros dansés)}

En 11) GIGUE DOUBLE, danseurs : MM. Israël et Olivier Dagenais, Guimbardiste : Mme Dagenais-Major.

Il s'agit sûrement de la Gigue de l'aveugle de la page 88 du programme. Notée en $2 / 4$, on n'indique rien sur la danse.

GIGUE CARRÉE, Danseurs : La famille Dagenais, Violoneux : M. Ménard Bougie

Serait-ce lié au reel à quatre de la page 89 ?

À la page 86, Barbeau écrit : « Il est impossible, à présent, de dire si les "gigues simples" et les "gigues doubles" sont vraiment différentes des "reels" écossais, du moins quant à l'origine des airs de violon qui les accompagnent. » On comprend donc que les deux types de gigue semblent s'exécuter sur le même genre de mélodies, qui auraient une consonance écossaise. Quant à la danse elle-même, on n'en sait pas plus.

À la page 86 , on nous présente le « Le Cotillon des dames », dont la partition est en 6/8. Aucune description de la danse, sinon que cela se danse avec quatre hommes et quatre femmes (en carré ? en colonne de couples ?). Page 88 : «Cotillon du Père Jarry », partition en 6/8, rien sur la danse. Notons au passage que le terme cotillon semble être utilisé de façon générique par Barbeau et certains de ses contemporains. La correspondance de Massicotte avec Barbeau nous le suggère par cette phrase :

[...] tout d'abord le numéro des grandes danses ou contredanses est difficile à établir en quelques semaines. Après divers essais je me suis arrêté au Spannedai [sic], la danse populaire des gaspésiens. J'ai trois couples de la Gaspésie qui l'exécutent avec brio. Cette contredanse que vous connaissez sans doute, tient du cotillon et de la gigue, et elle a belle allure. (Barbeau à Massicotte, 25-10-1920)

Le Spandy est donc présenté comme une contredanse tenant du cotillon, ce qui semble de prime abord contradictoire. Mais cela suggère que le terme cotillon prend ici un sens fort générique pour tout type de danse de figures, et expliquerait que des $2 / 4$ et des $6 / 8$ s'y trouvent regroupés, ainsi qu'une contredanse avec pas, comme le Spandy. Le cotillon serait-il l'équivalent 
du rigodon d'après-guerre, terme passe-partout pour différentes danses et diverses mélodies ? C'est ce que le programme des Veillées du bon vieux temps et la correspondance de Massicotte suggèrent.

À la page 85, Barbeau écrit :

Il y avait des danses et des airs de danse de différentes sortes. Ces danses se partagent aujourd'hui en deux groupes : les danses françaises et les danses anglaises. Les Canadiens-français ont assimilé très facilement un nombre considérable de danses anglaises, surtout par l'intermédiaire des Écossais et des Irlandais.

Barbeau suggère-t-il ici que les Écossais et les Irlandais présents au Québec pratiquaient principalement des danses anglaises ? Ou assimile-t-il tout simplement les répertoires écossais et irlandais à celui de l'Angleterre ? Ce n'est pas clair...

Il donne ensuite plusieurs rondes chantées comme exemples des danses françaises, puis poursuit :

Parmi les danses dont l'origine doit être française, il s'en trouvait d'autres sortes.

Citons : « La belle Catherine », « Les foins », « La frégate » (généralement appelée frégade ou frégarde), «La plongeuse », « Les cotillons », « Les quadrilles », et « Les menuets ».

La Belle Catherine est un classique écossais qu'on retrouve dans les recueils de Neil Gow dès 1788, et qui sera popularisée à travers toute l'Amérique du Nord. La Plongeuse pourrait bien être plutôt écossaise par son « dip \& dive », ou américaine, mais rien n'indique une origine française (on n'a jamais recueilli de contredanse de type whole set en France).

L'origine précise du cotillon québécois n'est pas encore fixée. Bien qu'il y ait certains liens avec les cotillons du début XVIII ${ }^{\mathrm{e}}$ siècle français, nos cotillons ont également beaucoup de ressemblances avec les Cotillions britanniques ou américains. Barbeau s'est-il simplement fié au nom « français » de la danse (à l'opposé de Money Musk, de Spandy, ou de Sir Roger de Coverley) pour assumer une origine française ?

\section{Programme du 29 avril 1920}

8) Danse populaire : Gigues et autres danses « La Varsovienne », le « Buck Reel ». Danseurs : MM. Isaïe et Honoré Leroux (84 et 86 ans, du Coteau-du-Lac, de Cornwall), Accordéoniste : M. Joachim Simard (de Québec), Guimbardiste : M. Louis Leduc.

On reste étonné par cette Varsovienne à deux hommes... Le Buck Reel (ou Buck \& Reel) est clairement identifié aux États-Unis comme une forme de clogging ou de tap dancing, et serait donc lié à la gigue.

12) Danses chantées: Rondes (La Boulangère, Ramenez vos moutons).

M. Philippe Lambert (originaire de Louiseville). 


\section{La recherche en danse au début du $\mathrm{Xx}^{\mathrm{e}}$ siècle}

Quel type de recherche en danse faisait-on au début du $\mathrm{Xx}^{\mathrm{e}}$ siècle, et où avaitelle principalement lieu? En Europe de l'Ouest, c'est l'Angleterre, où étudiait Marius Barbeau, qui fait figure de pionnière dans le domaine de la collecte des danses de figures (avant la Première Guerre). Cecil Sharp en est le chef de file, et fonde 1'English Folk Dance Society en 1911, qui fusionnera avec l'English Folk Song Society en 1932, pour donner l'English Folk Dance and Song Society. Entre 1931 et 1952, la France lancera une vaste réflexion sur les danses d'Europe et du monde en créant les Archives internationales de la danse (Paris), laboratoire scientifique et patrimonial, fondé par le mécène suédois Rolf de Maré dans le contexte de l'émergence de l'école française d'ethnologie.

Cecil Sharp publie en 1909 ses collectes de danses effectuées dans plusieurs villages d'Angleterre, avec descriptions fort détaillées des pas, positions et figures. Tout cela se passe donc un an avant que Barbeau ne dépose sa thèse à Oxford. Sharp ira ensuite collecter dans les Appalaches (Virginie, Caroline du Nord, Kentucky, Tennessee) lors de la Première Guerre, et publiera ses résultats de collectes en 1918.

La danse traditionnelle n'est pas un objet d'étude au début du $\mathrm{Xx}^{\mathrm{e}}$ siècle, sauf peut-être pour le pionnier qu'est Cecil Sharp. Bien que Barbeau fasse référence à Sharp dans son programme de 1928, ainsi que dans ses notes de cours de 1945, il ne semble pas avoir été influencé par Sharp pour développer un intérêt pour la danse.

Barbeau s'est aussi appuyé sur Madeleine Doyon-Ferland dans les années 1950. Dans l'introduction de la section « Fredons de danse et rondes » d'En roulant ma boule, Barbeau traite principalement des rondes et s'en remet ainsi à $\mathrm{M}^{\mathrm{me}}$ Doyon-Ferland pour les autres types de danses. Il reprend par exemple son affirmation : « [...] le brandy, dont l'origine est le branle français $^{6} »$. Assertion qu'on retrouve encore de nos jours dans L'Encyclopédie de la musique au Canada, à l'article " Danse - Musique utilisée avant 1867 » exprimée ainsi : « le brandy - autre variante de la danse du branle (bransle, brantle ou brawl, du verbe branler). ${ }^{7} \gg$ Rapprocher une danse en cercle de la Renaissance avec une contredanse giguée typique du XIX siècle pour la seule raison qu'elles commencent toutes deux par « bran... » est plutôt cocasse, pour le milieu du $\mathrm{Xx}^{\mathrm{e}}$ siècle, moins au $\mathrm{XXI}^{\mathrm{e}}$.

6. Marius Barbeau, En roulant ma boule, op. cit., p. 314 : « Traduction d'un extrait de Doyon [Ferland], Journal of American Folk-Lore, vol. 63, n² 248, 1950, p. 171-174. »

7. Helmut Kallmann (1922-2012), Gilles Potvin (1923-2000), Kenneth Winters (1929-2011), Encyclopédie de la musique au Canada, Toronto, Historica Foundation of Canada, 2001 ; en ligne : www.encyclopediecanadienne. ca/fr/article/danse-musique-utilisee-avant-1867. 
Barbeau est donc représentatif de son époque, c'est-à-dire de la période 1920-1950, qui ne portait guère d'attention à la danse comme champ d'étude. On pourrait rêver de ce qu'il aurait pu collecter comme danses dans les années 1920 s'il avait subi l'influence bénéfique de Cecil Sharp lors de son séjour en Angleterre. Mais ce ne fut pas le cas, et il faudra attendre le milieu des années cinquante pour voir Simonne Voyer faire les premières enquêtes dans le domaine.

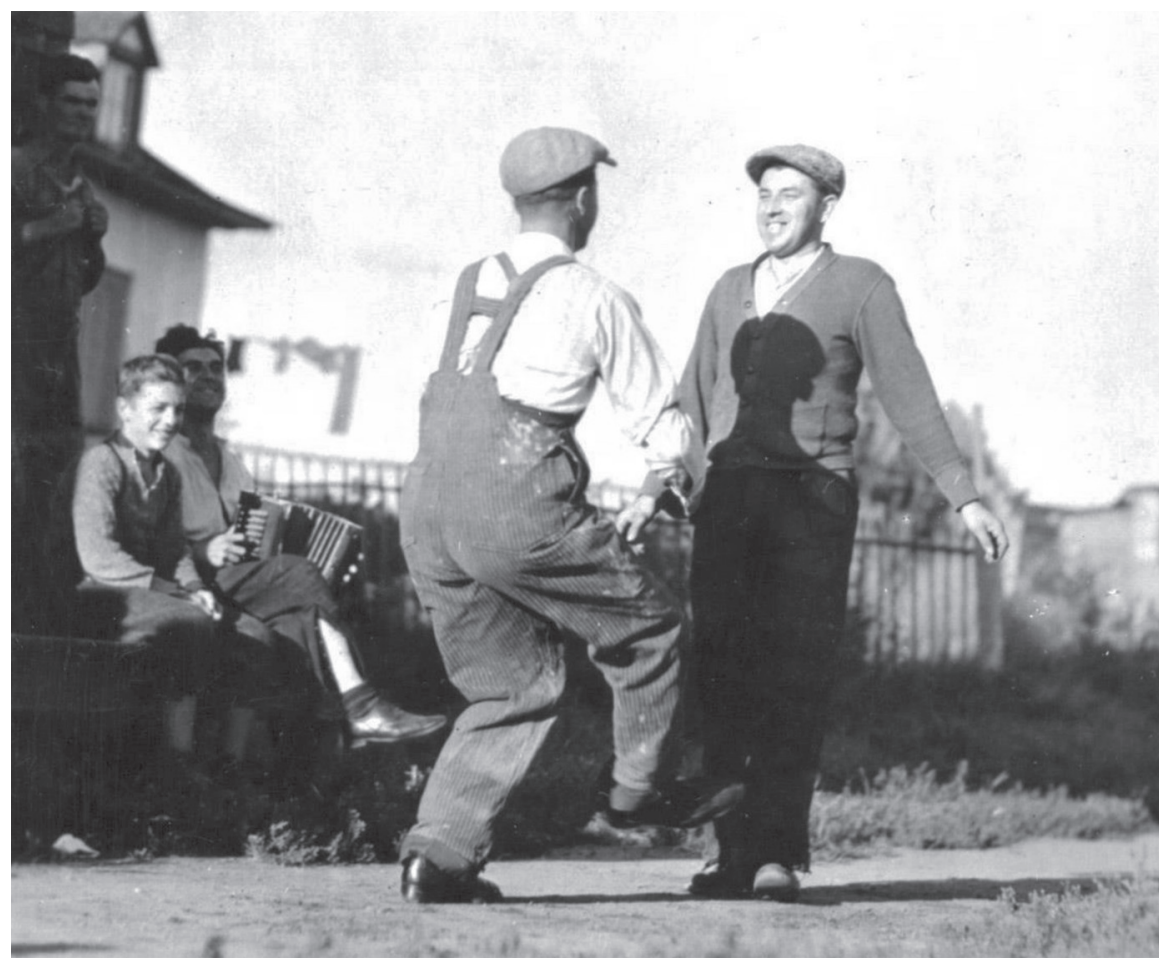

Danseurs près de Charlesbourg, Québec, 1941.

Source : Musée canadien de l'histoire no J299. 